\title{
Motor-Mediated Microtubule Self-Organization in Dilute and Semi-Dilute Filament Solutions
}

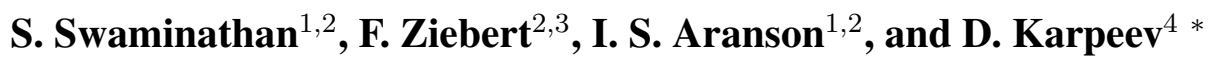 \\ ${ }^{1}$ Department of Engineering Sciences \& Applied Mathematics \\ Northwestern University, Evanston, IL 60208-3125 USA \\ ${ }^{2}$ Materials Science Division, Argonne National Laboratory, Argonne, IL, 60439 \\ ${ }^{3}$ Laboratoire de Physico-Chimie Théorique - UMR CNRS 7083, \\ ESPCI, 10 rue Vauquelin, F-75231 Paris, France \\ ${ }^{4}$ Mathematics \& Computer Science Division, Argonne National Laboratory, Argonne, IL, 60439
}

\begin{abstract}
We study molecular motor-induced microtubule self-organization in dilute and semidilute filament solutions. In the dilute case, we use a probabilistic model of microtubule interaction via molecular motors to investigate microtubule bundle dynamics. Microtubules are modeled as polar rods interacting through fully inelastic, binary collisions. Our model indicates that initially disordered systems of interacting rods exhibit an orientational instability resulting in spontaneous ordering. We study the existence and dynamic interaction of microtubule bundles analytically and numerically. Our results reveal a long term attraction and coalescing of bundles indicating a clear coarsening in the system; microtubule bundles concentrate into fewer orientations on a slow logarithmic time scale. In semi-dilute filament solutions, multiple motors can bind a filament to several others and, for a critical motor density, induce a transition to an ordered phase with a nonzero mean orientation. Motors attach to a pair of filaments and walk along the pair bringing them into closer alignment. We develop a spatially homogenous, mean-field theory that explicitly accounts for a force-dependent detachment rate of motors, which in turn affects the mean and the fluctuations of the net force acting on a filament. We show that the transition to the oriented state can be both continuous and discontinuous when the force-dependent detachment of motors is important.
\end{abstract}

Key words: microtubule, molecular motor, stochastic, fokker-planck, mechanics, collision, kinetic, bifurcation, weakly nonlinear analysis, pattern formation, molecular dynamics, active temperature, multiplicative noise, brownian motion.

\footnotetext{
*Corresponding author. E-mail: s-swaminathan@northwestern.edu
} 
AMS subject classification: 35Q82, 35Q84, 35Q92, 70F35, 82C26, 82C80

\section{Introduction}

Polar biofilaments, such as microtubules and actin, are known to form complex structures via interaction with molecular motors. Some examples include the formation of the cytoskeleton [15] and the assembly of mitotic spindles, which are used by eukaryotic cells to segregate chromosomes during cell division [17]. In each of these instances, microtubules undergo motor mediated attachment, cross-linking, and sliding which ultimately leads to the formation of highly organized structures [35]. The dynamics and self-organization of the cytoskeleton [27] is an important subject of study in the biological sciences as well as in soft matter physics as a natural realization of an out-of-equilibrium, complex fluid.

Pattern formations and intrinsic nonequilibrium dynamics inside the cytoskeleton have been investigated both experimentally [16, 29, 30, 37, 40, 41] and theoretically [1, 2, 24, 26, 44, 43]. In highly dilute filament solutions, binary interactions between motors and filaments are dominant. However, in the semi-dilute regime multiple motors can bind a filament to several other filaments. When a critical motor density is reached, these interactions induce a transition to an ordered phase with a nonzero mean orientation of filaments - the isotropic-polar transition - with the final state exhibiting coherent structures. A complete description of the dynamics remains a formidable task, since it involves nonequilibrium processes, intrinsic nonlinearities, structural anisotropies, and a broad window of time and length scales over the range of densities. For low filament densities, models based on binary rod interactions have been proposed [1,2, 26, 44], but in reality the cytosol is not sufficiently dilute to justify this approach. Additionally, hydrodynamic models have been formulated [20,34], but their validity is questionable due to the lack of scale separation between the patterns formed and the filament size. Moreover, the connections between the phenomenological parameters and the underlying microscopic mechanisms are not yet known. In small-scale simulations [37], interaction of rod like filaments by means of motor binding has been studied, and patterns resembling experimental ones have been observed. In [23], a model including transport of molecular motors along microtubules and motor-induced microtubule alignment was proposed. Simulations showed that asters and vortices form in this model.

In this paper, we discuss theoretical approaches used to model dilute and semi-dilute, spatially homogeneous filament solutions $[2,5,38,39]$. We further introduce methods for extending the models to spatially inhomogeneous regimes. In the dilute region, we begin by studying the nonlinear dynamics of Aranson's and Tsimring's [2] spatially homogeneous master equation given that the range of angular interaction is small. This limit is interesting because simulations of single motor-induced alignment have shown the onset of many localized bundles [43]. Our analysis of bundle coarsening dynamics shows that bundles have exponentially weak attraction accompanied by coalescing.

In the semi-dilute regime, we address alignment dynamics prevalent in motor-filament systems in which the filament density is too high for two-particle interaction models to be valid but still below the isotropic-nematic transition occurring for systems of passive anisotropic particles [31]. 
We use a Landau-Lifshitz type equation [22] and in our modeling take advantage of knowledge stemming from recently developed models for the alignment of two filaments [18, 45]. Specific effects that are taken into account include angle-dependent noise (due to intrinsic motor force fluctuations) and force-dependent detachment of motors. Our main result highlights the importance of the motor attachment dynamics in classifying the type of isotropic-polar transition; specifically, the inclusion of a force-dependent detachment rate makes a discontinuous ordering transition possible. This is in contrast to the usual phase transitions in two-dimensional nematics, which are second order as can be seen from the symmetry arguments of Landau and De Gennes [9]. Our work also illustrates the importance of the intrinsic fluctuations of motor forces (or "effective temperature") on the macroscopic behavior of motor-filament solutions. We account for the fact that the corresponding effective temperature exceeds the thermodynamic temperature by at least one order of magnitude and that therefore the motor-induced fluctuations should be more important than the thermal fluctuations. In principle, due to its multiplicative nature, motor fluctuations can also promote ordering of filaments for some parameter ranges (in contrast to thermal fluctuations).

This paper is organized as follows. The first part of our analysis is focused on the dilute solution binary interaction models. In section 2., we recall the spatially homogeneous rod alignment model proposed in [2], derive a limiting case for small angle interactions, and analyze unstable perturbations to the uniform state. In section 3. we discuss the framework for the asymptotic analysis of bundle interaction. The results of the asymptotics are compared with numerical experiments.

In the second part of the paper, self-organization in semi-dilute filament solutions is addressed. In section 4., we discuss the motor-filament interaction rules and develop the governing equations for the spatially homogeneous, motor-mediated self-organization of microtubules. In section 5. we show that discontinuous ordering transitions exist when motor detachment dynamics are important. We conclude in section 6 . with a discussion of results and open questions.

\section{Binary interaction model}

In the model introduced by Aranson and Tsimring [1], rods of orientation $\theta$ are aligned through irreversible pairwise interactions. These motor-mediated inelastic interactions are treated as instantaneous collisions in which each rod changes its orientation according to the following collision rule:

$$
\left(\begin{array}{c}
\theta_{1}^{f} \\
\theta_{2}^{f}
\end{array}\right)=\left(\begin{array}{cc}
\gamma & 1-\gamma \\
1-\gamma & \gamma
\end{array}\right)\left(\begin{array}{c}
\theta_{1}^{i} \\
\theta_{2}^{i}
\end{array}\right),
$$

where $\theta_{1,2}^{i}$ are the two rods' orientations before collision and $\theta_{1,2}^{f}$ are the orientations after. The constant $\gamma$ characterizes the inelasticity of collisions (analog of the restitution coefficient in granular media). The angle between two rods is reduced after collision by an "inelasticity" factor $\epsilon=2 \gamma-1$. Purely inelastic collisions correspond to $\gamma=\frac{1}{2}$ or $\epsilon=0$. In this analysis, we assume that two rods interact only if the angle between the rods is smaller than $\theta_{0}$; this requirement, after incorporating $2 \pi$-periodicity in alignment, gives the following interaction criteria: $\left|\theta_{2}^{i}-\theta_{2}^{i}\right|<\theta_{0}<\pi$ and $2 \pi-\theta_{0}<\left|\theta_{2}^{i}-\theta_{2}^{i}\right|<2 \pi$. Hence, rods collide inelastically through the aforementioned interaction rules, each rod acquiring the average orientation $\theta_{1}^{f}=\theta_{2}^{f}=\frac{\theta_{1}^{i}+\theta_{2}^{i}}{2}$. Rods are further subject to 
rotational diffusion due to random thermal fluctuations.

Consider $P(\theta, t)$ to be the probability distribution function of rods with orientation $\theta$ at time $t$, with normalizing condition, $\int_{-\pi}^{\pi} P d \theta=1$ Then, the master equation governing the spatially homogeneous self-organization of microtubules (derived in [1]) is given by,

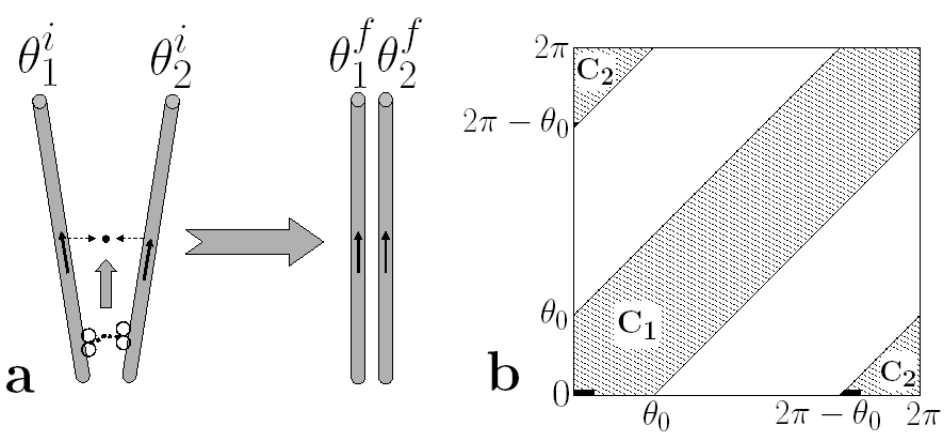

Figure 1: (a) Depiction of motor mediated microtubule interaction and alignment for fully inelastic collisions. (b)Angular interaction regions for colliding filaments

$$
P_{t}=D P_{\theta \theta}+g \int_{-\theta_{0}}^{\theta_{0}}\left[P\left(\theta+\frac{\omega}{2}\right) P\left(\theta-\frac{\omega}{2}\right)-P(\theta) P(\theta-\omega)\right] d \omega,
$$

where $\omega=\theta_{2}-\theta_{1}$. The "collision" integral in (2.1) accounts for interaction with gains associated with rods aligning to obtain the orientation, $\theta$, and losses due to rods having orientation $\theta$ before alignment.

In the case of interactions occurring only between rods initially nearly aligned, one can consider $\theta_{0}$ to be small. This limit is employed in order to study the formation and subsequent dynamics of microtubule bundles. For small $\theta_{0}$, the collision integral in equation (2.1) can be calculated via Taylor expansion of $P$ about $\omega=0$ to yield,

$$
\begin{aligned}
\int_{-\theta_{0}}^{\theta_{0}}\left[P\left(\theta-\frac{\omega}{2}\right) P\left(\theta+\frac{\omega}{2}\right)-P(\theta) P(\theta-\omega)\right] d \omega= \\
\quad \int_{-\theta_{0}}^{\theta_{0}}\left[-\omega P P^{\prime}-\frac{\omega^{2}}{4}\left(P P^{\prime \prime}+P^{\prime 2}\right)+\omega^{4}\left(\frac{-7}{192} P^{\prime \prime \prime \prime} P-\frac{1}{48} P^{\prime} P^{\prime \prime \prime}+\frac{1}{64} P^{\prime \prime 2}\right)\right] d \omega .
\end{aligned}
$$

Computing the integral in equation (2.2) and rearranging the derivatives in $\theta$ yields a nonlinear PDE which we refer to as the small angle master equation:

$$
P_{t}(\theta)=D \frac{d^{2} P}{d \theta^{2}}-g \frac{\theta_{0}^{3}}{12}\left[\frac{d^{2}}{d \theta^{2}}\left(P^{2}\right)+\frac{\theta_{0}^{2}}{80}\left(7 \frac{d^{4}}{d \theta^{4}}\left(P^{2}\right)-24 \frac{d^{2}}{d \theta^{2}}\left[\left(\frac{d P}{d \theta}\right)^{2}\right]\right)\right] .
$$

The disordered state, consisting of all equiprobable filament orientations, is represented by $P=$ $P_{0}=\frac{1}{2 \pi}$. Linear stability analysis about the uniform state reveals the unstable angular fourier 
modes $k$ as a function of the physical parameters. The band of unstable wave modes is given by,

$$
|k|<\sqrt{\frac{80}{\theta_{0}^{2}}\left[1-\frac{6 D}{7 g P_{0} \theta_{0}^{3}}\right]} .
$$

The parameters used in all of the numerical and analytical treatments to follow are, $g=100$, $D=0.1, \theta_{0}=0.5, P_{0}=\frac{1}{2 \pi}$. Substituting these parameters into (2.4) indicates that wave numbers of $k \approx 18$ and smaller yield instability in the linear limit.

The steady state small angle master equation can be evaluated both analytically as well as numerically. First, we introduce the scalings,

$$
\tilde{t}=t\left(\frac{10 D}{3 \theta_{0}^{2}}\right) ; \quad \tilde{\theta}=\theta\left(\sqrt{\frac{10}{3}} \frac{1}{\theta_{0}}\right) ; \quad \tilde{P}=P\left(\frac{\theta_{0}^{3} g}{12 D}\right),
$$

and write down the dimensionless small angle master equation (tildes are removed for brevity.),

$$
P_{t}(\theta)=\frac{d^{2} P}{d \theta^{2}}-\frac{d^{2}}{d \theta^{2}}\left(P^{2}\right)-\frac{7}{24} \frac{d^{4}}{d \theta^{4}}\left(P^{2}\right)+\frac{d^{2}}{d \theta^{2}}\left[\left(\frac{d P}{d \theta}\right)^{2}\right] .
$$

Sample long time numerical states are shown in Figure 2 below; these results show the existence of localized bundles of well aligned rods. $P, \theta$, and $t$ axes in all of the figures are scaled subject to $(2.5)$ (i.e. $0<\theta<2 \pi$ becomes $0<\theta<2 \pi\left(\sqrt{\frac{10}{3}} \frac{1}{\theta_{0}}\right)$ ). An implicit steady state analytical solution to (2.6) can also be calculated and was found in [38].

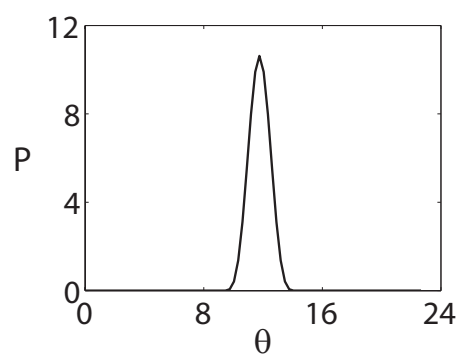

(a)

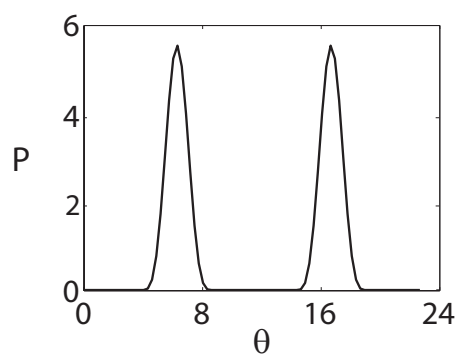

(b)

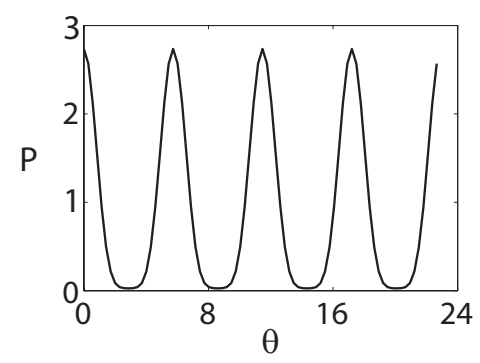

(c)

Figure 2: Typical numerically calculated long time bundle states computed from the small angle master equation. The initial conditions are the uniform density $P=1 / 2 \pi$ plus small perturbations of the form: (a) $\epsilon \cos \theta$, (b) $\epsilon \cos 2 \theta$, (c) $\epsilon \cos 4 \theta$. Equation (2.4) indicates that each of these initial conditions are unstable in the linear limit. The chosen parameters are, $g=100, D=0.1, \theta_{0}=$ $0.5, P_{0}=\frac{1}{2 \pi}, \epsilon=0.01$. 


\section{Bundle interaction dynamics}

Numerical analysis reveals that after the initial exponential growth of the instability, a well-defined multi-bundle configuration emerges. The intermediate stage follows, characterized by movement and alignment of bundles which ultimately exhibit highly nonlinear interactions leading to attractive motion. The final stages corresponding to bundles coalescing involve complicated dynamics which are not considered in this work.

In this study, we address the intermediate dynamics by preparing a two bundle initial state composed of two copies of a single bundle steady state configuration. This preparation is convenient because it allows us to skip the dynamics associated with shape change and focus on the movement. We expect, as indicated by our numerical studies, a quantifiable attracting interaction between bundles (refer to Figure 3). One can derive an analytical asymptotic expression for the

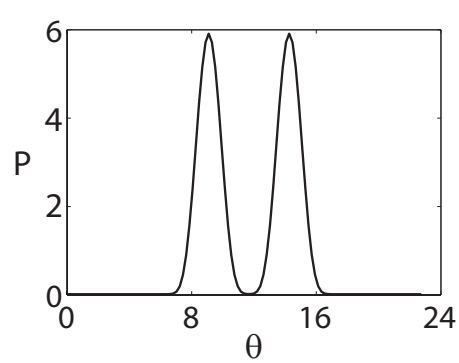

(a)

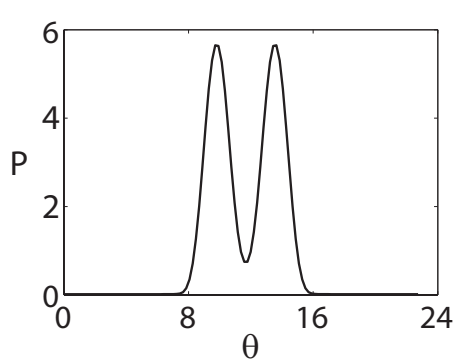

(b)

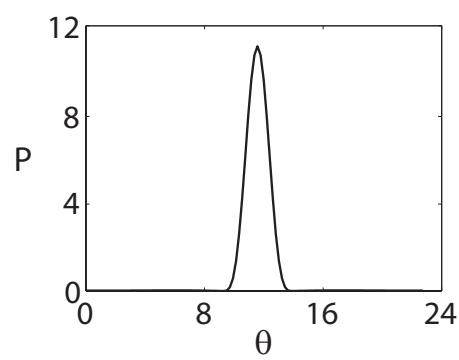

(c)

Figure 3: Numerical results showing the time evolution and eventual coalescing of two bundles of well aligned rods of different orientation:(a) Initial state, (b) Coalescing bundles, (c) Newly formed single bundle. The data indicates that the intermediate dynamics of bump interaction associated with movement is characterized by long term attraction.

time evolution of the distance between two bundles situated far from one another. In this large separation limit, the sum of two bundles can be considered an approximate steady state solution or leading order asymptotic steady state solution to (2.3). Through an application of the Fredholm Alternative one can derive an expression governing the rate at which bundles move toward one another. This procedure is outlined in exhaustive detail in [38]. The resulting expression is,

$$
X=\frac{1}{k} \ln \left|c_{1} k t+e^{k X_{0}}\right|
$$

where $X$ is the relative distance between two bundles. Figure 4(a) below shows the time evolution of the relative distance between two bundles as they move toward each other; Figure 4(b) shows the calculated value of $c_{1}$ for various $\tilde{\theta}_{0}$. The growth of $c_{1}$ with larger $\tilde{\theta}_{0}$ in Figure $4(\mathrm{~b})$ is consistent with the notion that larger $\tilde{\theta}_{0}$ yields greater interaction. Figure 4(c) shows the time evolution of an initial set of many bundles. The data in Figure 4(c) is fit to a function of the form, $\left(1+\frac{A}{\ln |B t+C|}\right)$, 
with fitting parameters $A, B, C$. This form was chosen because we know that the number of bundles scales as $N \sim \frac{L}{X}$, where $L$ is the domain length and $X$ is the distance between bundles; the distance between bundles changes logarithmically with time as indicated by (3.1).

The coalescing of bundles observed in simulations of two bundle and multi-bundle configurations indicates that coarsening in the system occurs on a slow logarithmic time scale. This finding is well illustrated in figures 4(a) and 4(c). Similar techniques for studying coarsening have been used in the study of axial segregation of granular materials. In [3], the authors show that bands of granular material coarsen on a similar logarithmic scale. Likewise, in [11], the authors find slow coarsening when studying the dynamics of slurries in circular and square tubes. When studied in complex, higher dimensional geometries, bands of segregated material have been known to exhibit slow coarsening but with more complicated dynamics [12]. Long term bundle formation is known to occur in liquid crystal systems as well; however, ordering in such systems is due mostly to thermodynamic considerations as opposed to active motor mediated effects [9].

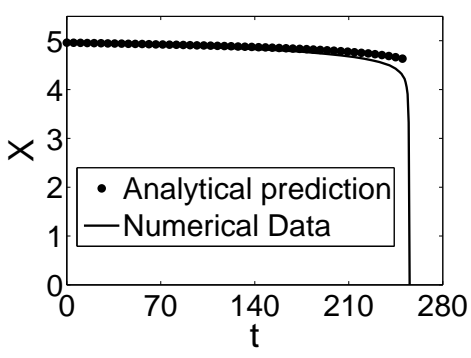

(a)

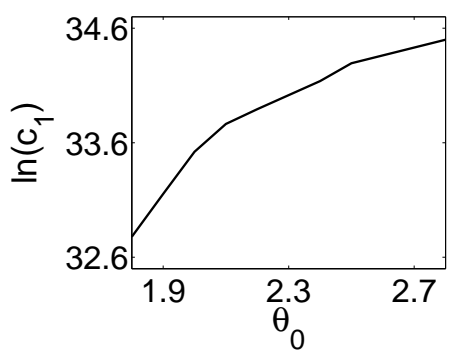

(b)

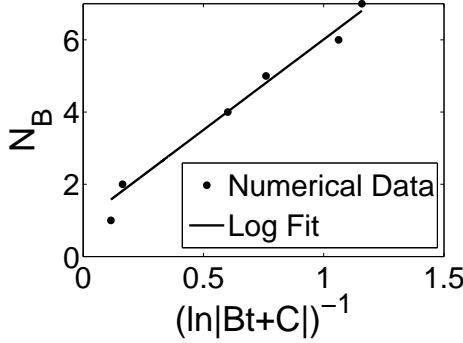

(c)

Figure 4: (a) Comparison between the numerical and analytical results for the time evolution of the relative distance between bundles; (b) $c_{1}$ as a function of $\theta_{0} . c_{1}$ represents the decay rate in the relative distance $X$; the growth of $c_{1}$ with increasing $\theta_{0}$ indicates that bundles coalesce more easily when a larger range of interaction angles are accepted; (c) Time evolution of the number of bundle orientations; the fit parameters are: $A=5.01, B=1.94, C=-1.50$.

\section{Semi-dilute solution model formulation \& associated Fokker- Planck equation}

Analysis in the previous sections was centered on bundle dynamics in dilute filament systems. In the semi-dilute case, microtubules (or short actin filaments) interacting via molecular motors are again modeled as a collection of $N$ stiff rods of fixed length $L$. For the sake of simplicity (and since most experiments are carried out in a quasi-two-dimensional geometry) we restrict our modeling to two spatial dimensions; the orientation of filament $i$ can then be described by a unit vector $\boldsymbol{\tau}_{i}=\left(\cos \varphi_{i}, \sin \varphi_{i}\right)$, or alternatively by the angle $\varphi_{i}$ (with respect to the $x$-axis, see 


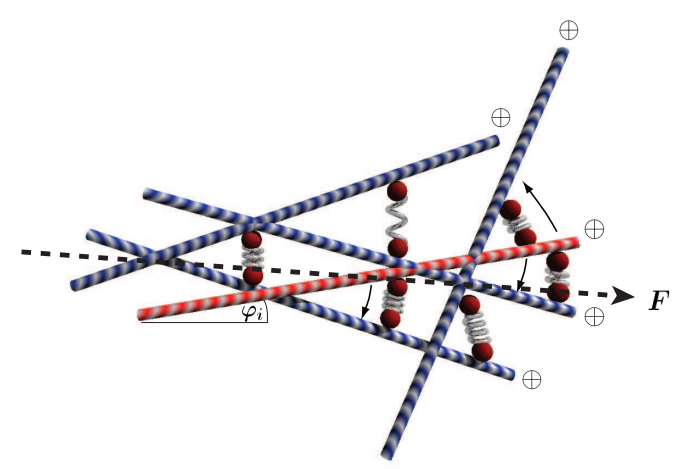

(a)

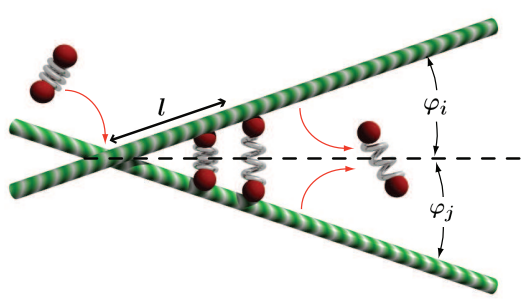

(b)

Figure 5: (a) Multi-rod configuration showing the interaction of the $i$-th microtubule (marked in red) with all other microtubules in the system. The dotted line indicates the direction of forcing due to all binary interactions with the $i$-th rod. (b) Depiction of motor mediated, binary microtubule interaction. Molecular motors attach at the rod intersection point, zip across the length of the rods causing alignment, and detach.

Figure 5(a)). The Landau-Lifshitz model [22], originally developed to describe the precessional motion of the magnetization in a ferromagnetic solid and augmented by Gilbert [13], captures the damped rotational dynamics prevalent in our system. In Gilbert's work, A vector representing the magnetization $\boldsymbol{M}$ in a magnetic field $\boldsymbol{H}$ moves so as to minimize $|\boldsymbol{M} \times \boldsymbol{H}|$, which leads to the equation:

$$
\dot{\boldsymbol{M}}=-\nu \boldsymbol{M} \times \boldsymbol{H}-\chi \boldsymbol{M} \times(\boldsymbol{M} \times \boldsymbol{H}),
$$

for some scalars $\nu$ and $\chi$. Accordingly, in our case a rod of orientation $\tau$ relaxes toward a field $\boldsymbol{F}$, which, coupled with the inextensibility requirement (the length of the rod is conserved), leads to the following equation of motion for the $i$-th rod:

$$
\zeta_{r} \dot{\boldsymbol{\tau}}_{i}=-\boldsymbol{\tau}_{i} \times\left(\boldsymbol{\tau}_{i} \times l_{m} \boldsymbol{F}\right)-\boldsymbol{\tau}_{i} \times \boldsymbol{T}
$$

where $\zeta_{r}$ is the rotational drag coefficient and $l_{m}$ is the average motor run length.

The mean field $\boldsymbol{F}$ represents the average force exerted on the $i$-th filament due to interactions with all other filaments. We intend to study the case of high motor density; hence, for a homogeneous filament solution, each rod is in motor contact with every other rod, and the total number of rods, $N$, is fixed. $\boldsymbol{T}$ is a thermal noise term given by,

$$
\boldsymbol{T}=\xi_{0} \hat{z} .
$$

Here $\xi_{0}$ is the stochastic torque and $\hat{z}$ is the unit vector perpendicular to the plane of the system. The stochastic force has zero mean, $\left\langle\xi_{0}\right\rangle=0$, (brackets represent the ensemble average), and is assumed to be $\delta$-correlated,

$$
\left\langle\xi_{0}(t) \xi_{0}\left(t^{\prime}\right)\right\rangle=2 k_{B} T \zeta_{r} \delta\left(t-t^{\prime}\right)
$$


where $k_{B} T$ is the thermal energy. The stochastic torque, as defined above, gives rise to rotational diffusion with diffusion coefficient $D_{r}=\frac{k_{B} T}{\zeta_{r}}$.

For the field $\boldsymbol{F}$ we use the following form,

$$
\boldsymbol{F}=\sum_{j=1}^{N} \boldsymbol{\tau}_{j}\left(\sigma \alpha+\xi_{j}\right),
$$

which represents the sum over all $(i, j)$ pairwise interactions (refer to Figure 5(a)); in the absence of spatial variations, this amounts to $N-1$ interactions. $\alpha$ is the average forcing strength for a single motor and $\sigma$ is the number of motors per filament pair. The stochastic term $\xi_{j}$ describes intrinsic fluctuations in the motor forces and enters as multiplicative noise.

During rod alignment, motors spanning the $i$-th and the $j$-th filaments stretch as they move away from the filament intersection point, exert a force on the $i j$ pair, and experience a corresponding restoring force. When motors attach, they quickly obtain a symmetric orientation perpendicular to the bisecting line of any two interacting rods, see Figure 5(b). This is due first to the fact that we assume negligible bending rigidity in the spring force which is supported by experimentation [30]. Second, there is a well accepted force-velocity relation $[6,15,19]$ given by,

$$
V_{M}=V\left(1-\frac{F_{l}}{F_{s t}}\right)
$$

where $V_{M}$ is the motor head velocity, $F_{l}$ is the projection of the motor spring force on the direction of the rod, and $F_{s t}$ is the so-called stall force. Thus, if a motor attaches asymmetrically, the leading motor head (corresponding to the larger distance from the filament intersection point) is slowed down by the force due to extension of the motor spring while the trailing head is accelerated, leading to fast relaxation towards a symmetric configuration. The force-velocity relation further implies that motors stop when the pulling force (or load) reaches the value of $F_{s t}$.

The number of motors $\sigma_{i j}$ spanning a filament pair takes the form,

$$
\sigma_{i j}=\sigma_{0} \exp \left(-\frac{\kappa a l\left|\sin \left(\frac{\varphi_{i}-\varphi_{j}}{2}\right)\right|}{k_{B} T}\right),
$$

where $\sigma_{0}$ is the average number of motors per microtubule. The exponential form in Eq. (4.5) is motivated by experimental studies [8] and was developed in Refs. [14, 32]; The argument of the exponential represents the ratio of the motor stretching energy to the thermal energy $k_{B} T$. The stretching of the motor is expressed by the average distance $l$ (along the microtubule) between the motor position and the intersection point of the two filaments (see Figure 5(b)) and by the angle between the filaments. $\kappa$ is the motor spring constant, known to be of the order of $200-400 \mathrm{pN} / \mu \mathrm{m}$ [7] for kinesin motors, and $a$ is a molecular length scale (a few $\mathrm{nm}$ ).

The noise terms occurring in the motor force, Eq. (4.4), are necessary to describe fluctuations in non-equilibrium systems of active motors that can potentially induce an effective temperature larger than the equilibrium (thermodynamic) temperature [24, 25, 28, 43]. Experiments in actinmyosin mixtures [28] showed strong deviations from thermodynamic equilibrium behavior due to 
motor fluctuations. We assume $\left\langle\xi_{i}(t)\right\rangle=0$ and

$$
\left\langle\xi_{i}(t) \xi_{j}\left(t^{\prime}\right)\right\rangle=2 \frac{\mu \zeta_{r}}{l_{m}^{2}} \delta\left(t-t^{\prime}\right) \delta_{i j}
$$

for $i, j=1 . . N$. The noise amplitude is comprised of $\mu=k_{B} T_{a}$, where $T_{a}$ is the effective (or active) temperature. The effective temperature can be estimated in terms of the mean motor force $\langle F\rangle$, motor step length $\langle L\rangle$, and $k_{B}$ as $T_{a}=\frac{\langle F\rangle\langle L\rangle}{k_{B}}$. Using known experimental values for kinesin - average motor force, $\langle F\rangle=5 \mathrm{pN}$ and average motor step length, $\langle L\rangle=8 \mathrm{~nm}$ [42] - we estimate the effective temperature to be $\simeq 3000 \mathrm{~K}$ (approximately 10 times room temperature), which is consistent with $[28,43]$.

If we write the orientation vector as, $\boldsymbol{\tau}_{i}=e^{i \varphi_{i}}$ and note the identities: $\boldsymbol{\tau}_{i} \times \boldsymbol{\tau}_{j}=-\sin \left(\varphi_{i}-\varphi_{j}\right) \hat{z}$ and $\boldsymbol{\tau}_{i} \times \hat{z}=-i \boldsymbol{\tau}_{i}$, where $\hat{z}$ is the unit normal to the plane, the equation of motion can be written in the following compact Langevin-type form:

$$
\zeta_{r} \dot{\varphi}_{i}=\alpha \sum_{j=1}^{N} \sigma_{i j} f_{i j}+\sum_{k=0}^{N} f_{i k} \xi_{k},
$$

with $f_{i j}=-l_{m} \sin \left(\varphi_{i}-\varphi_{j}\right)$ and $f_{i 0}=1$.

Since we assumed Gaussian white noise, this generalized Langevin equation is stochastically equivalent to a Fokker-Planck equation for the $N$ particle probability density $P^{(N)}(\vec{\varphi})$ with $\vec{\varphi}=\left(\varphi_{1}, \varphi_{2}, \ldots \varphi_{N}\right)$. We interpret the stochastic differential equation (4.7) in the Stratonovich sense. This is the natural interpretation if one assumes, as usually is the case in physical systems, a high frequency cut-off for the spectral density of the noise (see e.g. [33] for a discussion of multiplicative noise and Ito vs. Stratonovich interpretations). The Fokker-Planck equation for the probability density is,

$$
\begin{aligned}
& \frac{\partial}{\partial t} P^{(N)}(\vec{\varphi})=\frac{k_{B} T}{\zeta_{r}} \sum_{i, j=1}^{N} \frac{\partial^{2}}{\partial \varphi_{i} \partial \varphi_{j}} P^{(N)}(\vec{\varphi}) \\
& +\frac{\alpha l_{m}}{\zeta_{r}} \sum_{i, j=1}^{N} \frac{\partial}{\partial \varphi_{i}}\left[\sigma\left(\varphi_{i}-\varphi_{j}\right) \sin \left(\varphi_{i}-\varphi_{j}\right) P^{(N)}(\vec{\varphi})\right] \\
& +\frac{\mu}{\zeta_{r}} \sum_{i, j}^{N} \frac{\partial}{\partial \varphi_{i}}\left[\sin \left(\varphi_{i}-\varphi_{j}\right) \frac{\partial}{\partial \varphi_{j}}\left(\sin \left(\varphi_{i}-\varphi_{j}\right) P^{(N)}(\vec{\varphi})\right)\right]
\end{aligned}
$$

A mean field approximation is applied by assuming independence of rods as follows:

$$
P^{(N)}\left(\varphi_{1}, \ldots, \varphi_{N}\right)=P\left(\varphi_{1}\right) P\left(\varphi_{2}\right) \cdots P\left(\varphi_{N}\right)
$$

where

$$
P(\varphi)=P^{(1)}(\varphi)=\int_{-\pi}^{\pi} P^{(N)}\left(\varphi, \varphi_{2}, \ldots, \varphi_{N}\right) d \varphi_{2} . . d \varphi_{N}
$$


is the single-particle distribution function. This formulation is convenient because it converts a many bodied system into a single body problem.

We now integrate Eq. (4.8) with respect to the last $N-1$ angle variables. After rescaling $t \mapsto\left(\tilde{t} \zeta_{r}\right) / k_{B} T$, and defining $\tilde{\alpha}=\frac{\alpha l_{m}(N-1)}{k_{B} T}, \tilde{\mu}=\frac{\mu(N-1)}{2 k_{B} T}, \tilde{\sigma}=\sigma_{0} \sigma$ and $\tilde{\kappa}=\frac{\kappa a l}{k_{B} T}$, we obtain the mean-field equation for $P(\varphi)$ (In the following, tildes on scaled quantities are suppressed for brevity):

$$
\begin{aligned}
\frac{\partial}{\partial t} P(\varphi) & =\frac{\partial^{2}}{\partial \varphi^{2}} P(\varphi) \\
& +\alpha \frac{\partial}{\partial \varphi} \int \sigma\left(\varphi-\varphi^{\prime}\right) \sin \left(\varphi-\varphi^{\prime}\right) P(\varphi) P\left(\varphi^{\prime}\right) d \varphi^{\prime} \\
& -\mu \frac{\partial}{\partial \varphi}[(\gamma \sin 2 \varphi-\delta \cos 2 \varphi) P(\varphi)] \\
& +\mu \frac{\partial^{2}}{\partial \varphi^{2}}[(1-\gamma \cos 2 \varphi-\delta \sin 2 \varphi) P(\varphi)]
\end{aligned}
$$

We have introduced $\gamma=\int \cos 2 \varphi P(\varphi) d \varphi$ and $\delta=\int \sin 2 \varphi P(\varphi) d \varphi$ as abreviations for the second moments of $P(\varphi)$. The rotational drag coefficient is estimated in [10] as, $\zeta_{r} \simeq \frac{\pi \eta L^{3}}{3 \ln (L / b)}$, where $L=10-15 \mu \mathrm{m}$ is the microtubule length, $b=24 \mathrm{~nm}$ is the microtubule diameter, and $\eta \simeq$ $0.005 \mathrm{pNs} / \mu \mathrm{m}^{2}$ is the solvent viscosity. The time scale associated with thermal fluctuations is computed to be, $K_{B} T / \zeta_{r} \simeq 3 \cdot 10^{-3} \mathrm{~s}^{-1}$.

Equation (4.9) is a nonlinear and nonlocal PDE governing the time evolution of the probability density of filament orientations, $P(\varphi)$. It is related to the binary interaction models of Ref. [2, 21] in its incorporation of rotational diffusion due to thermal effects (given by the first term on the right hand side) and motor-induced interactions (proportional to the renormalized motor-strength $\alpha$ ). However, previous models assumed a priori binary interactions, whereas Eq. (4.9) is effectively quadratic in $P$ explicitly because of the approximation of independence of particles. Moreover, the derivation used here is unique in that it allows straightforward inclusion of the fluctuations in motor force. These are represented by the terms proportional to the renormalized fluctuation strength $\mu$, which should in fact be more important than the thermal effects for realistic conditions.

The uniform density corresponding to $P(\varphi)=P_{i s o}=\frac{1}{2 \pi}$, solves Eq. (4.9) upon application of the normalization condition, $\int_{0}^{2 \pi} P(\varphi) d \varphi=1$. It represents an isotropic distribution of rods. If motor affects are sufficiently stronger than diffusion associated with thermal fluctuations, the uniform state loses its stability resulting in the onset of spontaneous orientation. In [39], the formation of oriented states is studied in two physical cases. The first case is that in which the motor detachment dynamics are negligible (ie: for $\sigma=\sigma_{0}$ ). In this case, one can perform weakly nonlinear analysis in a parameter space near the instability threshold. These calculations lead to the Landau-type equation for the time evolution of the mean orientation (respresented by the first Fourier mode). Analysis of the Landau equation indicates that the ordering transition is always second order (or continuous) in agreement with numerical analysis. In the following section, we address the general case studied in [39] in which detachment dynamics are relevant. This analysis is interesting because it shows that force-dependent motor detachment and intrinsic motor fluctuations can shift the ordering transition from second order to first order. 


\section{Influence of force dependent detachment rate}

One can gain some insight into the dynamics of the master equation given by Eq. (4.9) by examining its terms. The exponential form of Eq. (4.5), for example, indicates that the strength of motorinduced interactions is greatly diminished when the angle between interacting rods is large. Hence, one would anticipate that the intermediate ordering dynamics would involve multiple steady state rod bundles of disparate orientation similar to those found and studied extensively in the binary interaction models previously presented. Furthermore, one would expect a more complex transition to alignment due to the nonlocal, motor-induced alignment term, i.e. the term proportional to $\alpha$ in Eq. (4.9).

The general master equation is highly nonlinear, and the convolutions in the integral term indicate that analysis is more convenient in Fourier space. Consider the Fourier harmonics of $P(\varphi)$ given by,

$$
P(\varphi)=\frac{1}{2 \pi} \sum_{n} P_{n} e^{i n \varphi}, \quad P_{n}=\int_{0}^{2 \pi} P(\varphi) e^{-i n \varphi},
$$

Substituting Eq. (5.1) into Eq. (4.9) yields equations for the Fourier coefficients $P_{n}$ :

$$
\begin{aligned}
\dot{P}_{n}= & -n^{2} P_{n}(1+\mu) \\
& +\frac{1}{2} \alpha n \sum_{k} P_{n-k} P_{k}\left(q_{k-1}-q_{k+1}\right) \\
& -\frac{\mu n}{2}\left(P_{n-2} P_{2}-P_{n+2} P_{-2}\right) \\
& +\frac{\mu n^{2}}{2}\left(P_{n-2} P_{2}+P_{n+2} P_{-2}\right)
\end{aligned}
$$

where we have defined,

$$
q_{k}=\frac{1}{2 \pi} \int_{0}^{2 \pi} \exp \left[-\kappa\left|\sin \left(\frac{\varphi}{2}\right)\right|-i k \varphi\right] d \varphi .
$$

One can identify the unstable angular wave modes near the isotropic state by linearizing Eq. (5.2) about the uniform density corresponding to $P=P_{0}$. The growth rate of the $n$-th Fourier mode, $\lambda_{n}$, is found to be,

$$
\begin{array}{ll}
|n| \neq 2: & \lambda_{n}=\frac{\alpha n}{2}\left(q_{n-1}-q_{n+1}\right)-n^{2}(1+\mu) \\
|n|=2: & \lambda_{ \pm 2}=\alpha\left(q_{\mp 1}-q_{ \pm 3}\right)-4-3 \mu .
\end{array}
$$

Eqs. (5.4) and (5.5) indicate that several modes may lose stability depending on the magnitude of the motor strength parameter $\alpha$, the values of the $q_{k}$, and the motor noise strength $\mu$. This was not the case for the limit of small detachment rate.

The type of ordering transition can be determined by deriving a Landau-type equation using the following procedure: Expand $\mathrm{P}$ as, 


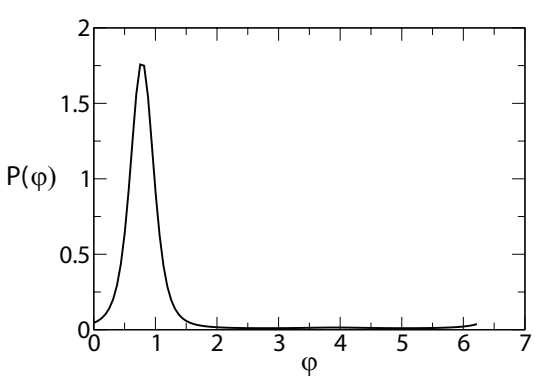

(a)

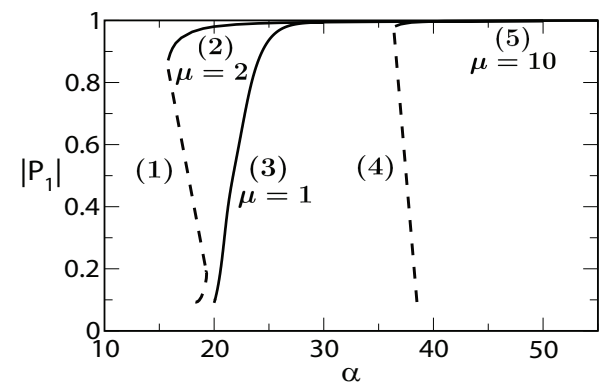

(b)

Figure 6: (a) Steady state distribution obtained from numerical treatment of (4.9) with parameters: $\alpha=16 \mu=2$. (b) Bifurcation diagram showing the stable states with nonzero mean orientation associated with the supercritical (3) and subcritical (2) transitions from the isotropic state. The stable branch (5) was calculated for the limiting case of zero diffusion. The dotted branches (1) and (4) show estimates of the repelling branches associated with the subcritical transitions.

$$
P(\varphi, t)=\frac{1}{2 \pi}\left(1+\epsilon \tilde{P}_{1}\left(\varphi, t_{\epsilon}\right)+\epsilon^{2} \tilde{P}_{2}\left(\varphi, t_{\epsilon}\right)+\ldots\right)
$$

where,

$$
t_{\epsilon}=\epsilon^{2} t
$$

and

$$
\tilde{P}_{n}=P_{n}\left(t_{\epsilon}\right) e^{i n \varphi}+P_{-n}\left(t_{\epsilon}\right) e^{-i n \varphi} .
$$

Keeping terms up to $O\left(\epsilon^{2}\right)$ and solving for $P_{1}$ yields the familiar Landau-type equation,

$$
\begin{aligned}
\dot{P}_{1} & =\Lambda_{1} P_{1}-A\left|P_{1}\right|^{2} P_{1}+R, \\
A & =\frac{\alpha^{2}\left(q_{0}-q_{2}\right)\left(q_{-2}-q_{0}+q_{1}-q_{3}\right)}{2\left[\alpha\left(q_{-1}-q_{3}\right)-4-3 \mu\right]}, \\
\Lambda_{1} & =\frac{\alpha}{2}\left(q_{0}-q_{2}\right)-1-\mu,
\end{aligned}
$$

where $R$ represents higher order regularizing terms. These have to be included in the subcritical case $A<0$, where the lowest order nonlinearity of the Landau equation is insufficient to get amplitude saturation. They can be calculated but are not specified here. In Eq. (5.9), both the instability threshold $\Lambda_{1}>0$ and the nonlinear Landau coefficient governing whether the transition is supercritical $(A>0)$ or subcritical $(A<0)$, depend on the values of the $q_{k}$. They have to be computed approximately using known parameter estimates. We have $\tilde{\kappa}=\frac{\kappa a l}{k_{B} T}$. The motor spring constant $\kappa$ has been measured to be $200-400 \mathrm{pN} / \mu \mathrm{m}$ for kinesin motors [7]. For the molecular 
length scale $a$, one usually assumes a few $\mathrm{nm}[14,32]$. The parameter $l$, which represents the average distance from the point of motor attachment to the filament intersection can be estimated to be about 100nm [45]. This indicates that $\frac{\mathrm{kal}}{k_{B} T} \simeq 5-10$ at room temperature; we use a value of 5.1 to compute the $q_{k}$-terms and write down the simplified Landau coefficients as,

$$
\begin{aligned}
A & =\frac{1.28 \cdot 10^{-3} \alpha^{2}}{2(0.352 \alpha-4-3 \mu)} \\
\Lambda_{1} & =0.176 \alpha-1-\mu .
\end{aligned}
$$

The coefficient $A$ changes sign depending on the relationship between the motor-induced alignment strength $\alpha$ and the motor noise $\mu$. This suggests that there exists a qualitative difference in the transition to alignment depending on the parameter selection, which is supported by numerical simulations of Eq. (4.9). Figure 6(a) shows a long time oriented state for $\alpha=16$ and $\mu=2$. The peak is steeper, i.e. the orientation is more perfect, as compared to that found in the small detachment rate case. This is caused by the subcriticality. Figure 6(b) shows both a continuous bifurcation as well as a subcritical bifurcation from the isotropic state for fluctuation strengths $\mu=1$ and $\mu=2$ respectively. As a common feature of subcritical transitions, the stable branch below the linear stability threshold indicates a type of hysteresis in which an oriented state exists below threshold, depending on the initial conditions. The subcritical transition for $\mu=10$ was calculated for the limiting case of zero thermal effects (i.e. rotational diffusion $D_{r}=k_{B} T / \zeta_{r} \rightarrow 0$ or $T \rightarrow 0$ ). As the motor forcing strength is known to be 3-4 times the noise amplitude [42], the following relationship holds for the renormalized parameters. $\alpha \simeq 3 \sqrt{\mu}$. We can thus conclude that the limit of vanishing thermal effects is always subcritical for reasonable parameters of $\kappa(1<\kappa<10), \alpha$, and $\mu$.

\subsection{Extensions to spatially inhomogeneous filament organization}

The mean field formulation presented in the previous sections can be extended to include spatially inhomogeneous motor-filament dynamics by analyzing the system's conserved quantities. The master equation given by (4.7), for example, is an expression of torque conservation. Specifically, motor-mediated torque is balanced by viscous drag effects. Inclusion of the respective force balance in the formulation (similar to the construct used in [2]) leads to a system of equations describing both the rotational and translational motion of each filament. Molecular dynamics type simulations can then be used to evolve these master equations and study structure formation. The details of this formalism are left for future discussion. Figure 7 below displays the output of a sample molecular dynamics simulation in which vortices arise arise from initially disordered filament systems. These patterns are in qualitative agreement with those found in in vitro experiments on self-organization in motor-filament systems [30,37]. A detailed analysis of the parameter space which leads to such patterns and an examination of the intrinsically fluctuating quantities (motor forces and thermal effects for example) are both important topics for future research. 


\section{Conclusions}

In this paper, we reviewed a filament alignment model proposed in [1] and modified in [38] which governs the self organization of microtubules for near alignment interactions. We found that initially disordered systems exhibit an ordering instability resulting in the onset of bundles of similarly oriented microtubules. The analysis of the coarsening dynamics associated with these filament bundle interactions was the focus of the study.

Our primary result was the derivation of an expression governing the relative motion of bundles. Both numerical simulations and asymptotic methods show that bundles initially situated far from one another exhibit attractive behavior. The subsequent coalescing shows a coarsening in the system as bundles become concentrated in fewer orientations. The time scale of coarsening in 2 bundle and multi-bundle configurations is shown to be logarithmic.

The asymptotic calculations governing the time evolution of the relative distance between bundles showed good quantitative agreement with numerical simulations. Deviations in the analytical work from the numerics occured only when bundles were nearly aligned. This is, of course, expected as the asymptotic analysis was known to be accurate only when applied to bundles at large relative distances. Further numerical simulations showed that the speed at which bundles move toward each other increased with increasing $\theta_{0}$. This finding was in qualitative agreement with simulations of the general alignment model proposed in [1].

We extended our analysis to semi-dilute filament solutions in which previous binary interaction models $[1,2,5,38]$ are insufficient. In this limit, we employed a mean field formulation in which any given filament interacts with all other filaments in motor contact. Both additive noise associated with thermal fluctuations and multiplicative noise describing small scale fluctuations in motor forcing were incorporated into this model. Finally, we accounted for force-dependence in the detachment kinetics of the motors. With this approach we found that an initially disordered system exhibits an ordering instability resulting in the onset of well aligned rod bundles; a finding

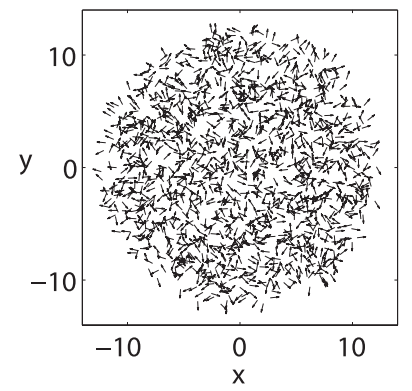

(a)

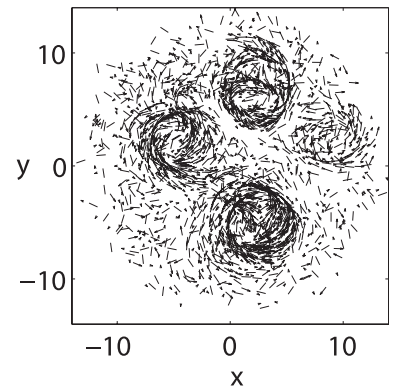

(b)

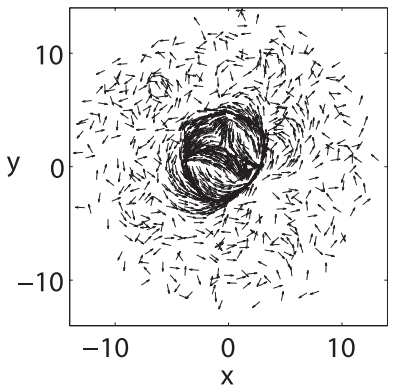

(c)

Figure 7: Select simulation results: (a)-(c) Molecular dynamics simulation of vortex formation and coalescence arising from an initially disordered filament system. Snapshots are taken at times (a) $t=0$, (b) $t=170$ and (c) $t=370$ on the time scale of rotational diffusion (refer to the time rescaling discussed in section 4.). 
that is consistent with previous binary interaction models.

The qualitative nature of filament ordering is studied via analysis of a Fokker-Planck equation derived from the mean field model for the alignment of rods. We showed that first and second order transitions to alignment exist when motor forcing dominates random forcing fluctuations. The existence of a subcritical branch is shown to be a function of the motor spring strength and the ratio of motor forcing to motor and thermal noises. At the moment we are not aware of experimental validations for the subcritical scenario. However, the model predicts ordering hysteresis for in vitro experiments on alignment dynamics in semi-dilute and dense solutions of biological filaments.

There are many open questions. First, we discussed an exact integral solution to the small angle master equation which was derived in [38]. We are yet to obtain a great deal of meaning from it, and further analysis could be useful, particularly in understanding the type, number, and long time behavior of the numerically determined bundle states. Second, in the semi-dilute case, the question of when and under which conditions the isotropic-to-polar transition is second or first order should be investigated and clarified experimentally. Finally, we briefly discussed vortex formation in simulations of spatially inhomogeneous microtubule self-organization, but we are yet to do any rigorous analytical work. With the inclusion of spatial inhomogeneity, one could explore the critical parameter space in which rods become disordered; we could then derive in a similar fashion to Ref. [2], a coupled system of Ginzburg-Landau equations in both the mean field and binary interaction limits. Additional molecular dynamics simulations could be used to investigate structure formations like bundles and asters.

Ultimately, although our analysis is problem specific, we believe that the concept of inelastic collisions in angle space resulting in alignment of rod directions is a primary mechanism driving self organization in many physical systems. A few examples include rod-shaped swimming bacteria, vibrated granular rod systems $[4,36]$, and solutions of charged nanorods. Further investigation and modifications of our models could provide deeper insight into many other physical processes.

The authors would like to thank Eli Ben-Naim, Vladimir Volpert, and the late Alexander Golovin (father, friend, and colleague) for useful discussions throughout the period of this study.

The work of I.S.A. and S.S. was supported by the U.S. Department of Energy, Office of Basic Energy Sciences, Division of Materials Science and Engineering, under the Contract No. DEAC02-06CH11357. This paper was revised and redrafted under the advisement of Monica Olvera De La Cruz with funding from the Northwestern University Nonequilibrium Research Center (NERC), Award No: DE-SC0000989. F.Z acknowledges funding by the German Science Foundation (DFG).

\section{References}

[1] I. S. Aranson, L. S. Tsimring. Pattern formation of microtubules and motors: Inelastic interaction of polar rods. Phys Rev E, 71 (2005), No. 5, 050901.1-050901.4.

[2] I. S. Aranson, L. S. Tsimring. Theory of self-assembly of microtubules and motors. Phys Rev E, 74 (2006), No. 3, 031915.1-031915.15. 
[3] I. S. Aranson, L. S. Tsimring, V. M. Vinokur. Continuum theory of axial segregation in a long rotating drum. Phys Rev E, 60 (1999), No. 2, 1975-1987.

[4] I. S. Aranson, D. Volfson, L. S. Tsimring. Swirling motion in a system of vibrated elongated particles. Phys Rev E, 75 (2007), No. 5, 051301.1-051301.9.

[5] E. Ben-Naim, P. L. Krapivsky. Alignment of rods and partition of integers. Phys Rev E, 73 (2006), No. 3, 031109.1-031109.6.

[6] O. Campas, Y. Kafri, K. B. Zeldovich, J. Casademunt, J. F. Joanny. Collective dynamics of interacting molecular motors. Phys Rev Lett, 97 (2006), No. 3, 038101.1-038101.4.

[7] C. M. Coppin, T. Finer, J. A. Spudich, R. D. Vale. Measurement of the isometric force exerted by a single kinesin molecule. Biophys. J., 68 (1995), 242s-244s.

[8] C. M. Coppin, D. W. Pierce, L. Hsu, R. D. Vale. The load dependence of kinesin's mechanical cycle. Proc. Natl. Acad. Sci., 94 (1997), No. 16, 8539-8544.

[9] P. G. de Gennes, J. Prost. The Physics of Liquid Crystals. Clarendon Press, Oxford, 1993.

[10] M. Doi, S. F. Edwards, The Theory of Polymer Dynamics, Clarendon Press, Oxford, 1986.

[11] S. J. Fiedor, J. M. Ottino. Dynamics of axial segregation and coarsening of dry granular materials and slurries in circular and square tubes. Phys Rev Lett, 91 (2003), No. 24, 244301.1244301.4.

[12] T. Finger, A. Voigt, J. Stadler, H. G. Niessen, L. Naji, R. Stannarius. Coarsening of axial segregation patterns of slurries in a horizontally rotating drum. Phys Rev E, 74 (2006), No. 3 , 031312.1-031312.15.

[13] T. L. Gilbert. A phenomenological theory of damping in ferromagnetic materials, IEEE Trans. Magn, 40 (2004), No. 6, 3443-3449.

[14] S. W. Grill, K. Kruse, F. Jülicher. Theory of mitotic spindle oscillations. Phys Rev Lett, 94 (2005), No. 10, 108104.1-108104.4.

[15] J. Howard. Mechanics of Motor Proteins and the Cytoskeleton, Springer, New York, 2001.

[16] D. Humphrey, C. Duggan, D. Saha, D. Smith, J. Käs. Active fluidization of polymer networks through molecular motors. Nature (London), 416 (2002), No. 6879, 413-416.

[17] L. C. Kapitein, E. J. G. Peterman,B. H. Kwok, J. H. Kim,T. M. Kapoor,C. F. Schmidt. The bipolar mitotic kinesin Eg5 moves on both microtubules that it crosslinks. Letters to Nature, 435 (2005), No. 7038, 114-118.

[18] D. Karpeev, I. S. Aranson, L. S. Tsimring, H. G. Kaper. Interactions of Semiflexible Filaments and Molecular Motors, Phys Rev E, 76 (2007), No. 5, 051905.1-051905.12. 
[19] S. Klumpp, R. Lipowski. Cooperative cargo transport by several molecular motors. Proc. Natl. Acad. Sci., 102 (2005), No. 48, 17284-17289.

[20] K. Kruse, J. F. Joanny, F. Jülicher, J. Prost, K. Sekimoto. Asters, vortices, and rotating spirals in active gels of polar filaments. Phys Rev Lett, 92 (2004), No.7, 078101.1-078101.4.

[21] K. Kruse, F. Jülicher. Self-organization and mechanical properties of active filament bundles. Phys Rev E, 67 (2004), No. 5 051913.1-051913.16.

[22] L. D. Landau, E. M. Lifshitz,On the theory of the dispersion of magnetic permeability in ferromagnetic bodies. Phys. Z. Sovietunion, 8 (1935), 153-169.

[23] H. Y. Lee, M. Kardar. Macroscopic equations for pattern formation in mixtures of microtubules and motors. Phys Rev E, 64 (2001), No. 5 056113.1-056113.8.

[24] D. Loi, S. Mossa, L. F. Cugliandolo. Effective temperature of active matter. Phys Rev E, 77 (2008), No. 5, 051111.1-051111.4.

[25] T. B. Liverpool, A. C. Maggs, A. Ajdari. Viscoelasticity of solutions of motile polymers. Phys Rev Lett, 86 (2001), No. 18, 4171-4174.

[26] T. B. Liverpool, M. C. Marchetti. Instabilities of isotropic solutions of active polar filaments. Phys Rev Lett, 90 (2003), No. 13, 138102.1-138102.4.

[27] H. Lodish, A. Berk, S. L. Zipursky, P. Matsudaira, D. Baltimore J. Darnell. Molecular Cell Biology, W.H. Freeman, New York, 1999.

[28] D. Mizuno, C. Tardin, C. F. Schmidt, F. C. MacKintosh. Nonequilibrium mechanics of active cytoskeletal networks. Science, 315 (2007), No. 5810, 370-373.

[29] F. Nédélec, T. Surrey, A. C. Maggs. Dynamic Concentration of Motors in Microtubule Arrays. Phys Rev Lett, 86 (2001), No. 14, 3192-3195.

[30] F. J. Nédélec, T. Surrey, A. C. Maggs, S. Leibler. Self-organization of microtubules and motors. Nature (London), 389 (1997), No. 6648, 305-308.

[31] L. Onsager. Effects of shape on the interaction of colloidal particles. Ann. N.Y. Acad. Sci., 51 (1949), No. 4, 627-659.

[32] A. Parmeggiani, F. Jülicher, L. Peliti, J. Prost. Detachment of molecular motors under tangential loading. Europhys Lett, 56 (2001), No. 4, 603-609.

[33] H. Risken. The Fokker-Planck Equation, Springer, Berlin (1989).

[34] R. A. Simha, S. Ramaswamy. Hydrodynamic fluctuations and instabilities in ordered suspensions of self-propelled particles. Phys Rev Lett, 89 (2002), No. 5, 058101.1-058101.4. 
[35] D. Smith, F. Ziebert, D. Humphrey, C. Duggan, M. Steinbeck, W. Zimmermann, J. Kas. Molecular motor-induced instabilities and cross-linkers determine biopolymer organization. Biophysical Journal, 93 (2007), No. 12, 4445-4452.

[36] A. Sokolov, I. S. Aranson, J. O. Kessler, R. E. Goldstein. Concentration dependence of the collective dynamics of swimming bacteria. Phys Rev Lett, 98 (2007), No. 15, 158102.1158102.4.

[37] T. Surrey, F. Nédélec, S. Leibler, E. Karsenti. Physical properties determining selforganization of motors and microtubules. Science, 292 (2001), No. 5519, 1167-1171.

[38] S. Swaminathan, D. Karpeev, I. S. Aranson. Bundle dynamics of interacting polar rods. Phys Rev E, 77 (2008), No. 6, 066206.1-066206.9.

[39] S. Swaminathan, F. Ziebert, D. Karpeev, I. S. Aranson. Motor-mediated alignment of microtubules in semidilute mixtures. Phys Rev E, 79 (2009), No. 3, 036207.1-036207.8.

[40] K. Takiguch., Heavy meromyosin induces sliding movements between antiparallel actin filaments. J. Biochem, 109 (1991), No. 4, 520-527.

[41] R. Urrutia, M. A. McNiven, J. P. Albanesi, D. B. Murphy, B. Kachar. Purified kinesin promotes vesicle motility and induces active sliding between microtubules in vitro. Proc Natl Acad Sci, 88 (1991), No. 15, 6701-6705.

[42] R. D. Vale, R.A. Milligan. The way things move: Looking under the hood of molecular motor proteins. Science, 288 (2000), No. 5463, 88-95.

[43] F. Ziebert, I. S. Aranson, Rheological and structural properties of dilute active filament solutions, Phys Rev E, 77 (2008), No. 1, 011918.1-011918.5.

[44] F. Ziebert, I. S. Aranson, L. S. Tsimring. Effects of crosslinks on filament-motor organization. New J. Phys., 9 (2007), No. 11, 421.1-421.14.

[45] F. Ziebert, M. Vershinin, S. P. Gross, I. S. Aranson. Collective alignment of polar filaments by molecular motors. Eur. Phys. J. E, 28 (2009), No. 4, 401-409. 